\title{
Gestion anténatale et issue postnatale des foetus atteints de laparoschisis
}

\author{
Prenatal care and postnatal outcome for fetuses with laparoschisis
}

\author{
X. Capelle ${ }^{\mathrm{a}}$, J.-P. Schaaps ${ }^{\mathrm{a}, \mathrm{b}}$, J.-M. Foidart ${ }^{\mathrm{a}}$

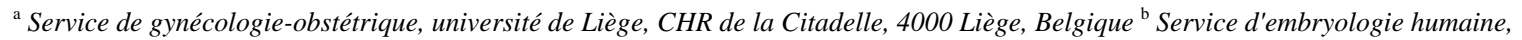 \\ université de Liège, Belgique
}

\section{Résumé}

Objectifs. - Évaluer la pertinence et la qualité de la prise en charge du laparoschisis, pathologie malformative rare, dans un centre de référence de moyenne importance. Matériel et Méthode. - Étude rétrospective portant sur 24 cas de laparoschisis pris en charge au Centre hospitalier régional de la Citadelle, département de gynécologie et d'obstétrique de l'université de Liège, entre 1992 et 2003 en confrontant les données anténatales aux données pédiatriques.

Résultats. - Vingt-quatre cas de laparoschisis ont été inclus. Le diagnostic prénatal a été posé dans 22 cas sur 24 à un âge gestationnel moyen de 23 semaines (92 \%). Aucune anomalie du caryotype ou malformation associée n'a été décelée en anténatal. Trois enfants avec une communication interauriculaire sans répercussion fonctionnelle et un syndrome du X fragile ont été diagnostiqués en postnatal. Une interruption médicale de grossesse a été réalisée à 24 semaines et une mort fœtale in utero est survenue à 35 semaines. Des lésions d'atrésie digestive de sténose ou d'ischémie sont présentes à la naissance chez huit enfants. Parmi les 22 enfants nés vivants, dix (45\%) ont pu bénéficier d'une cure chirurgicale primaire. Trois enfants sur 22 (14\%) ont nécessité une réintégration en deux temps sans complication également. Neuf enfants sur 22 (41\%) ont subi des interventions multiples (deux à six). Ceux-ci ont tous présenté des complications postnatales, parfois systémiques, incluant trois décès, six enfants avec complications infectieuses, cinq avec complications digestives et deux avec complications rénales ou hématologiques. La durée d'hospitalisation varie de 19 à 378 jours (médiane $=51$ jours). La durée du séjour hospitalier et le délai avant alimentation entérale totale sont allongés chez les enfants ayant présenté en prénatal un oligohydramnios ou des signes échographique de lésions digestives. Parmi les enfants nés avant 35 semaines, seuls ceux chez qui l'on constate à la naissance des lésions ischémiques digestives ont un séjour hospitalier et un délai avant alimentation entérale totale plus longs. Vingtdeux enfants sont nés vivants (92\%) et 19 ont survécu (86\%) après un an. Le taux de survie sans handicap corrélé au laparoschisis est de $84 \%$.

Conclusion. - L'échographie est une méthode valide de diagnostic et de surveillance anténatale. Nos résultats sont en accord avec les bons taux de survie actuellement rapportés dans la littérature. Il faut cependant souligner la longueur du séjour hospitalier et la fréquence des complications postopératoires. Le pronostic est essentiellement conditionné par la sévérité des lésions digestives ischémiques constatées à la naissance pour lesquelles les possibilités de diagnostic et de prise en charge anténatale sont actuellement réduites.

Mots clés : Laparoschisis ; Échographie obstétricale ; Résultats

\begin{abstract}
:
Objectives. - To assess the relevance and the quality of gastroschisis's care in a mid level referral centre. Method. - A retrospective analysis was performed for infants diagnosed or born with gastroschisis between 1992 and 2003 at the Citadelle hospital, Department of Obstetrics and Gynaecology, University of Liège. Results. - Twenty-four cases of gastroschisis were identified. For 22 of them (92\%) antenatal sonographic diagnosis was performed at a mean gestational age of 23 weeks. Antenatal diagnosis did not allow to identify additional malformation or chromosomal anomaly. Postnatal diagnosis allows to identify 3 infants with minor cardiac anomalies without functional consequence and one $\mathrm{X}$ fragile syndrome. One pregnancy was electively terminated at 24 weeks and one late intrauterine death was reported at 35 weeks. Bowel atresia, stenosis or ischemia were present at birth for 8 cases (33\%). Out of 24 cases 22 were live born. 10 infants out of 22 (45\%) underwent uncomplicated primary surgical repair. Three infants out of 22 (14\%) underwent delayed closure without complications. Nine infants out 22 (41\%) underwent multiple surgery (2 to 6). In this group all had postnatal complications, some with multisystem complications, including 3 deaths, 6 with infectious complications, 5 with gastrointestinal complications and 2 with genitourinary or haematological complications. Hospital stay range from 19 to 378 days (median, 51 days). Length of stay and time to full enteral feeding were longer if oligohydramnios or sonographic signs of intestinal damage were found. Among infants born before 35 weeks, only those with intestinal damage at birth had length of stay or time to full enteral feeding longer. Out of 22 live born infants 19 survived (86\%) after one year. Survival rate without handicap due to gastroschisis is $84 \%$.
\end{abstract}


Conclusion. - Sonographic examination is a valid method for prenatal diagnosis and surveillance. Our survival rate agrees with recent data in the literature. It has to be noticed that hospital stay is lentgthy and complications are frequent. The most important prognostic factor is the condition of the bowel at birth and there is no antenatal means to predict severe damage.

Keywords: Gastroschisis; Obstetric sonography; Surveillance

\section{INTRODUCTION}

Le laparoschisis est la malformation de la paroi abdominale le plus fréquemment rencontrée après l'omphalocèle.

La généralisation du diagnostic in utero par l'échographie depuis les années 1980 a permis de donner les informations nécessaires aux parents autrefois confrontés brutalement à cette pathologie lors d'une perte fœtale ou à l'accouchement.

Dans le même temps, les progrès de la réanimation néonatale et de la nutrition parentérale ainsi que l'apparition de matériel prothétique dans les traitements chirurgicaux ont largement contribué à l'amélioration spectaculaire du pronostic de ces enfants.

Cette évolution favorable est également redevable d'une nécessaire concertation multidisciplinaire basée sur la précision et la fiabilité du diagnostic anténatal. Celle-ci permettra de définir une conduite à tenir pour une prise en charge périnatale optimale.

Malgré ces évolutions, certaines controverses persistent concernant la définition et la pertinence des facteurs pronostiques échographiques, les avantages d'un accouchement prématuré et des interventions in utero ainsi que l'intérêt de la césarienne par rapport à la voie basse. Il a paru intéressant dans le cadre de la pratique liégeoise d'évaluer la pertinence et la qualité de la prise en charge d'une pathologie rare dans un centre de référence de moyenne importance permettant ainsi une évaluation indispensable à l'optimalisation des soins à la lumière des données récentes de la littérature.

\section{Étiopathogénie}

Le laparoschisis se définit comme une ouverture pariétale paraombilicale, habituellement droite, de 2 à $4 \mathrm{~cm}$ qui intéresse toutes les couches de la paroi abdominale. L'intestin éviscéré est toujours le siège d'une mal rotation et d'une absence de fixation secondaire à la paroi abdominale postérieure.

Le cordon ombilical est normalement inséré. Les anses intestinales sont directement exposées au liquide amniotique. D'autres organes peuvent être herniés, le côlon, l'estomac et plus rarement le système génitourinaire.

L'incidence dont l'augmentation depuis 30 ans laisse supposer l'implication de facteurs tératogènes environnementaux est de 1 pour 10000 naissances [1].

Il s'agit d'une malformation sporadique, le plus souvent isolée, non associée à des aberrations chromosomiques et touchant principalement les primipares jeunes dans les milieux socioéconomiques défavorisés [2].

Sur le plan du développement, c'est au cours de la quatrième semaine que l'embryon subit un phénomène complexe de délimitation qui a pour effet de convertir le disque embryonnaire tridermique plat en une structure à trois dimensions. De façon concomitante aux phénomènes de délimitation, on assiste à des modifications du système vasculaire : les veines ombilicales fournissent la vascularisation de la partie antérieure de la paroi abdominale avant d'être remplacées par les artères omphalomésentériques (branches de l'aorte). Durant la cinquième semaine, la veine ombilicale droite s'atrophie ainsi que l'artère omphalomésentérique gauche. L'artère omphalomésentérique droite formera le tronc cœliaque et l'artère mésentérique supérieure. Deux hypothèses étiopathogéniques existent.

La première implique une anomalie d'involution de la veine ombilicale droite qui serait responsable d'un déséquilibre de la balance entre la prolifération cellulaire et l'apoptose des éléments mésodermiques voisins impliqués dans la formation de la paroi abdominale [3,4] et conduirait au défect paraombilical droit observé. 
L'autre hypothèse implique un phénomène disruptif de l'artère omphalomésentérique droite qui permettrait d'expliquer les lésions digestives associées à la malformation [5]. Les théories embryologiques vasculaires sont en accord avec les données épidémiologiques impliquant l'usage de certaines substances vasoactives (alcool, cocaïne, tabac, pseudoéphédrine, acide acétylsalicylique) comme facteur de risque du laparoschisis [6].

\section{Physiopathologie des lésions digestives}

La physiopathologie des lésions digestives qui conditionnent le pronostic des enfants atteints de laparoschisis continue de faire débat. Trois processus ayant chacun leur dynamique spécifique peuvent être retenus :

- l'hypothèse selon laquelle un arrêt du développement des artères omphalomésentériques (dont dérivera l'artère mésentérique supérieure) est à l'origine du laparoschisis permet d'envisager certaines lésions intestinales ischémiques (sténose et atrésie) comme un trouble précoce du développement vasculaire ;

- une compression, éventuellement croissante durant la grossesse, des anses au niveau du collet de la malformation permet d'expliquer l'apparition de lésions ischémiques partielles ou complètes avec nécroses segmentates et perforation intestinales [7] ;

- il existe également des lésions d'ordre inflammatoires et tardives [8]. La présence des anses dans le liquide amniotique contenant des substances d'origine urinaire et digestive va induire une réaction inflammatoire. Celleci prend l'aspect d'une péritonite aseptique avec œdème, dépôt de fibrine et infiltrat cellulaire. Tout l'intestin peut être engainé dans cette périviscérite.

Différentes expérimentations animales ont permis de mieux cerner les mécanismes responsables de la périviscérite.

Langer et al. en 1989 [9] réalisent un laparoschisis chez des fœtus de mouton au jour 80 (terme = 145 jours.) en définissant quatre groupes. Un laparoschisis expérimental est créé par extériorisation des anses grêles à travers un anneau de silastic pour deux groupes dont un ou la mise en place d'un lien à la base de l'intestin hernié induit une compression croissante avec l'évolution de la gestation. Deux groupes subissent une laparotomie sans extériorisation des anses dont un groupe témoin et l'autre ou un lien est placé à la base de l'intestin grêle.

Les phénomènes de périviscérite sont observés uniquement en rapport avec le contact prolongé des anses dans le liquide amniotique. Les lésions intestinales (dilatation veineuse et lymphatique mésentérique, atrophies villositaires) sont le fait de l'ischémie induite par la mise en place du lien avec ou sans exposition au liquide amniotique. Il existe également un effet indépendant négatif du liquide amniotique et du lien sur la motilité intestinale testée in vitro en réponse à la stimulation par l'acétylcholine.

D'autres groupes ont utilisé l'embryon de poulet dont la séparation complète de la cavité amniotique et de la cavité allantoïde (contenant les produits d'excrétion urinaire et digestive à travers le cloaque) a permis de créer des modèles expérimentaux intéressants.

La réalisation d'un laparoschisis chez l'embryon de poulet ne s'accompagne de lésions de périviscérite que lorsque les intestins sont exposés au contenu de l'allantoïde par l'intermédiaire d'une brèche entre les deux cavités. Kluck et al. [10] estimaient donc en 1983 que les composants urinaires étaient responsables des phénomènes inflammatoires. Il constate que les lésions débutent au jour 15 (terme $=21$ ) et sont liées à une augmentation de la concentration amniotique en urée et en créatinine. Ces résultats sont en accord avec les observations de Tibboel sur des fœtus humains à différents moments de la grossesse : les lésions de périviscérite apparaissent après 30 semaines et peuvent être corrélées à des modifications qualitatives de l'urine fotale.

Les travaux d'Aktüg $[11,12]$ ont partiellement confirmé cette hypothèse. Le remplacement du liquide amniotique par une solution physiologique empêche la formation d'une périviscérite.

Ces travaux ont cependant démontré que l'amnioéchange influençait peu la présence d'éléments d'excrétion urinaire (urée et créatinine) en raison de leur production continue par l'embryon de poulet. Ce qui n'est pas le cas des sels biliaires et de la bilirubine dont les concentrations dans le liquide amniotique sont abaissées de façon durable après amnioéchange.

De plus, la création d'un laparoschisis au jour 5 (terme $=21$ ) sans effraction de l'allantoïde avec injection intraamniotique secondaire soit d'urine soit de méconium ne s'accompagne de périviscérite que dans le groupe 
méconium. En utilisant des solutions de différentes concentrations, Api et al. [13] ont pu montrer que la concentration intra-amniotique de méconium devait atteindre un certain niveau pour provoquer des lésions digestives.

C'est également dans ce cens que vont les travaux d'Akgür et al. dont les résultats reposent sur le modèle expérimental chez le rat adulte [14]. Un groupe de rats a reçu pendant cinq jours des injections intrapéritonéales biquotidiennes d'urine néonatale humaine dans des conditions aseptiques. L'autre groupe s'est vu injecter dans les mêmes conditions une solution à 5 \% de méconium de nouveau-né. L'examen anatomopathologique des anses intestinales après le sacrifice des rats au sixième jour montre, dans le groupe " méconium », des lésions identiques aux lésions observées en cas de périviscérite chez le nouveau-né humain porteur d'un laparoschisis. Les anses en contact avec l'urine ne montrent en revanche aucune lésion.

Ces données expérimentales impliquent clairement le méconium dans l'apparition des lésions et démontrent l'effet bénéfique de l'amnioéchange sur la périviscérite.

Les observations de défécation physiologique in utero et le pourcentage élevé de liquide amniotique méconial en cas de laparoschisis permettent dans une certaine mesure d'étendre ces conclusions à l'homme.

La périviscérite s'accompagne d'une inflammation intra-amniotique démontrée par les travaux de Morrison et al. [15] à partir d'échantillons de liquide amniotique prélevés au troisième trimestre en cas de laparoschisis. Ces échantillons montrent un exsudat inflammatoire aigu composé de macrophages et de monocytes activés d'origine principalement fœtale. L'interleukine 8 (inducteur puissant de la migration et de l'activation des macrophages) a été mesurée à des valeurs comparables à celles rencontrées dans les chorioamniotites infectieuses. L'interleukine 6, qui intervient notamment dans la cascade d'événements biologiques déclenchant le travail, est également élevée.

Ces travaux ont permis de construire un modèle cohérent de la physiopathologie de la périviscérite. Une péritonite aseptique chronique se constitue en raison du contact direct des anses digestives avec le liquide amniotique dans lequel elles baignent. Le méconium semble l'élément principalement impliqué dans ce phénomène. La périviscérite qui s'accompagne d'un exsudat inflammatoire et d'un véritable phénomène de drainage des protéines sériques du fœtus vers le compartiment amniotique est responsable d'un état de souffrance chronique. Le stress et la douleur causés par ces lésions entraînent une nette augmentation de la sécrétion de bétaendorphines fœetales dans les cas sévères [16].

Les médiateurs inflammatoires, et en particulier l'interleukine 6, libérés en grande quantité dans le liquide amniotique peuvent déclencher le travail prématurément. Luton et al. [17] ont montré qu'il existe une corrélation entre la sévérité de la périviscérite, le degré de prématurité et la longueur du séjour hospitalier (Fig. 1).

Figure 1: Laparoschisis présentant des lésions ischémiques sévères, malrotation digestive atrésie du côlon droit et microcôlon en aval.

Gastroschisis with sever ischemic damage, non rotated bowel with atresia and stenosis of large intestine.

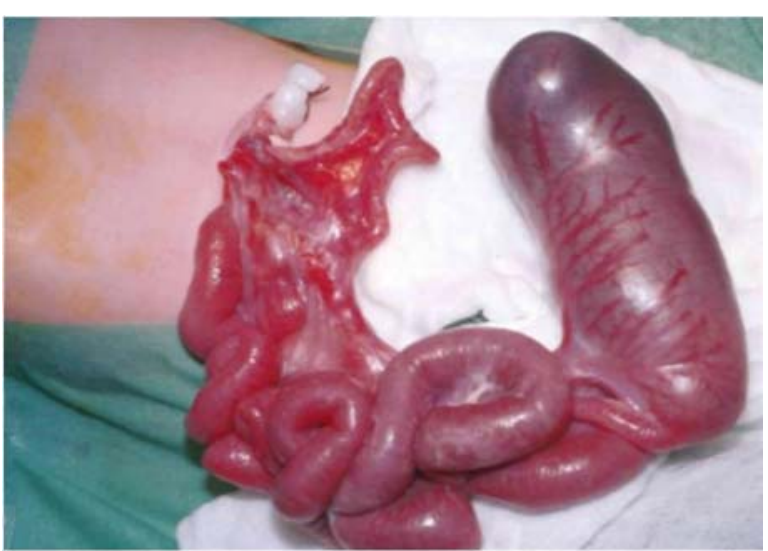

\section{MATERIEL ET METHODES}

L'analyse que nous avons réalisée porte sur les cas de laparoschisis diagnostiqués ainsi que ceux concernant les enfants ayant bénéficié d'un transfert in utero ou postnatal dans le service universitaire du Centre hospitalier régional de la Citadelle à Liège entre 1992 et 2003. La liste de ces cas $(n=24)$ a été constituée à partir des 
fichiers informatisés d'échographie obstétricale du service et du résumé clinique minimum en utilisant les codes de la classification internationale des maladies correspondants.

Les données ont été collectées rétrospectivement à partir des protocoles échographiques, du dossier obstétrical et pédiatrique. Les paramètres maternels enregistrés sont les suivants : âge gestité, âge gestationnel au moment du diagnostic, terme et voie d'accouchement ainsi que l'indication des césariennes. Soixante-dix-neuf protocoles d'échographies réalisés par les deux échographistes de référence du service ont été revus en considérant la quantité du liquide amniotique, les dilatations digestives, l'épaississement des parois intestinales, le doppler puisé des artères mésentériques, l'hyperéchogénicité des anses ou du liquide amniotique et la croissance fœtale. Ces données ont été confrontées au suivi pédiatrique.

Les données pédiatriques suivantes ont également été collectées : âge et poids de naissance, anomalies associées, modalités et délai de la cure chirurgicale (réparation primaire versus utilisation d'un silo prothétique), complications opératoires, délai avant alimentation entérale totale, durée du séjour hospitalier et évolution (Fig. 2).

\section{RESULTATS}

L'âge maternel moyen en début de grossesse est de 22 ans dont 14 patientes primigestes (58 \%). Le diagnostic échographique exact a été posé à un âge gestationnel moyen de 23 semaines dans 91 \% des cas. Une recherche de caryotype foetal a été proposée dans 21 cas, 19 résultats, tous normaux, ont été obtenus. Quinze fœtus sont de sexe féminin pour neuf de sexe masculin. Un des fœtus est issu d'une grossesse gémellaire monochoriale monoamniotique.

Figure 2 : Laparoschisis avec extériorisation digestive massive (estomac, cadre duodénal et intestin grêle) présentant une périviscérite agglutinant les anses intestinales.

Gastroschisis with important bowel protrusion (stomach duodenum and small intestine) and massive inflammatory coating reaction.

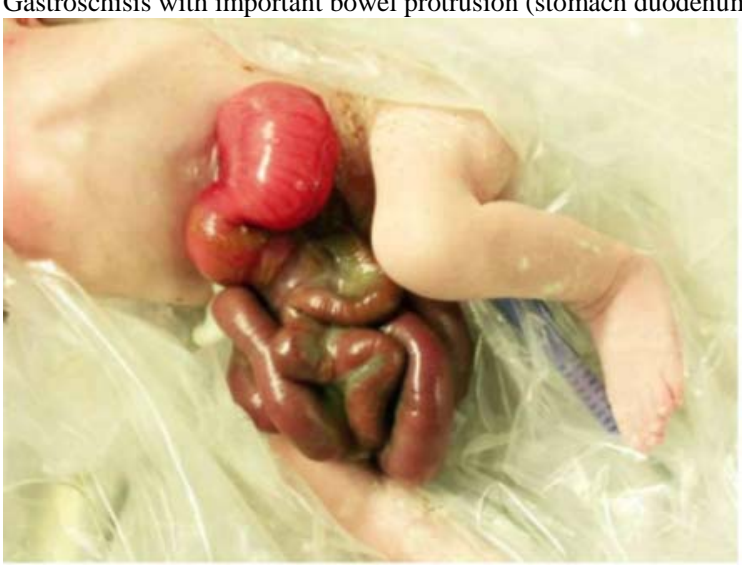

Un oligohydramnios a été diagnostiqué dans neuf cas sur 24 et un seul cas de polyhydramnios est relevé. Trois retards de croissance intra-utérine ont été diagnostiqués.

Vingt-deux enfants sont nés vivants, une interruption médicale de grossesse a été réalisée à 24 semaines et une mort fœtale in utero est survenue à 35 semaines. Onze enfants sur 22 sont nés par césarienne. Le liquide amniotique est méconial dans $40 \%$ des naissances.

Treize enfants sur 22 (59\%) sont nés avant 37 semaines dont deux avant 32 semaines. Dans quatre cas (18\%) le poids de naissance est inférieur au percentile 10. Le délai moyen entre la naissance et la prise en charge chirurgicale est de 2 heures 30 excepté pour un enfant pris en charge à l'extérieur et opéré le lendemain de sa naissance. Des lésions ischémiques ou d'atrésie digestive ayant nécessité un geste chirurgical ont été observées chez huit enfants (33\%). La durée d'hospitalisation varie de 19 à 378 jours (moyenne 75 jours, médiane 51 jours).

Un premier groupe concernant dix enfants (45\%) a pu bénéficier d'une réparation chirurgicale primaire (en un temps), la durée moyenne d'alimentation parentérale est de 31 jours et la durée d'hospitalisation de 35 jours. Dans le deuxième groupe, trois enfants (14\%) ont nécessité une réintégration en deux temps sans complication également, les durées moyennes d'alimentation parentérale (28 jours) et d'hospitalisation (46 jours) sont comparables. 
Le troisième groupe est constitué des neuf enfants (41\%) avec interventions multiples (deux à six) et complications opératoires (septicémie, endocardite, abcès de paroi et rejet de matériel prothétique, occlusion intestinale, insuffisance rénale aiguë, coagulation intravasculaire disséminée). Dans ce groupe, trois enfants sont décédés dans les trois premiers mois. Les durées moyennes d'alimentation parentérale (75 jours) et d'hospitalisation (95 jours) sont nettement augmentées pour les six enfants restants.

Tableau 1: Fréquence des malformations, des syndromes et des dyschromosomies associées aux laparoschisis en comparaison avec les données de Stoll et al., 2001, Barisic et al., 2001 et Poulain et al., 1994

Proportion of concurrent malformations syndromes or chromosomal abnormalities in gastroschisis in comparaison with Stoll et al., 2001, Barisic et al., 2001 and Poulain et al., 1994

\begin{tabular}{|c|c|c|c|c|}
\hline & CHR 1992-2003 & $\begin{array}{l}\text { Stoll et al., } 2001 \\
265858 \text { naissances }\end{array}$ & $\begin{array}{l}\text { Barisic et al., } 2001 \\
690123 \text { grossesses }\end{array}$ & $\begin{array}{l}\text { Poulainet al., } 1994 \\
764 \text { NN + laparoschisis }\end{array}$ \\
\hline$N$ Laparoschisis & 24 & 47 & 106 & 764 \\
\hline Prévalence/10 000 & & 1,76 & 1,54 & \\
\hline Formes isolées & $87,5 \%$ & $46,8 \%$ & $77 \%$ & $91,4 \%$ \\
\hline Formes associées & $12,5 \%$ & $53,2 \%$ & $23 \%$ & $8,6 \%$ \\
\hline \multirow[t]{4}{*}{ Dyschromosomies } & $0 \%$ & $2,1 \%$ & $2 \%$ & $0,6 \%$ \\
\hline & & \multirow[t]{3}{*}{1 Transloc. déséquilibrée } & 1 TRI. 13 & \multirow{4}{*}{$\begin{array}{l}2 \text { TRI. } 13 \\
2 \text { TRI. } 18 \\
1 \text { MONOS. } 22\end{array}$} \\
\hline & & & 1 TRI. 21 & \\
\hline & & & & \\
\hline $\begin{array}{l}\text { Malformations } \\
\text { diverses }\end{array}$ & 8,5 \% Cardiaque & $\begin{array}{l}\text { 46,8\% Gastro-int. } \\
\text { SNC }\end{array}$ & $14 \%$ & \\
\hline \multirow{3}{*}{$\begin{array}{l}\text { Syndromes non } \\
\text { chromosomiques }\end{array}$} & $4 \%$ & $4,2 \%$ & $7 \%$ & \multirow[t]{3}{*}{ Bandes amniotiques } \\
\hline & Sy. X Fra. & Limb body wall complex & Limb body Wall Complex & \\
\hline & & Squlettal dysplas. & Bandes amniotiques & \\
\hline
\end{tabular}

Tableau 2: Comparaison de l'évolution postnatale des cas de laparoschisis ayant présenté un oligohydramnios et les cas avec liquide amniotique normal en considérant au moins trois échographies à partir du diagnostic Comparaison between postnatal outcome for gastroschisis cases with oligohydramnios and those with normal amniotic fluid index

\begin{tabular}{lll}
\hline & Oligoamnios $(\boldsymbol{n}=\mathbf{9})$ & Liquide amniotique normal $(\boldsymbol{n}=\mathbf{6})$ \\
\hline Interventions & $16(1,8)$ & $11(1,8)$ \\
Délai < AET & 70 & 28 \\
Durée séjour & 95 & 52 \\
Enfants avec complications digestives & $2(0,2)$ & $3(0,5)$ \\
\hline
\end{tabular}

Délai < AET : délai en jours avant alimentation entérale totale.

Tableau 3 : Comparaison de l'évolution postnatale des cas de laparoschisis ayant présenté au moins un signe échographique péjoratif (dilatations des anses intestinales ou de l'estomac, épaississement des parois digestives, hyperéchogénicité digestive ou du liquide amniotique, diminution de la vascularisation mésentérique ou de la péristaltique) et les cas avec échographie non compliquée en considérant au moins trois echographies à partir du diagnostic

Comparaison between postnatal outcome for gastroschisis cases with at least one sonographic sign of intestinal damage and those with ultrasonographic examination reported as uncomplicated

\begin{tabular}{lll}
\hline & $\begin{array}{l}\text { Paramètres échographiques péjoratifs } \\
(\mathbf{n = 8 )}\end{array}$ & $\begin{array}{l}\text { Echographies non compliquées } \\
(\mathbf{n}=\mathbf{9})\end{array}$ \\
\hline Interventions & $16(2)$ & $13(1,6)$ \\
Délai < AET & $103^{\mathrm{a}}$ & $35^{\mathrm{b}}$ \\
Durée séjour & $127^{\mathrm{a}}$ & $53^{\mathrm{b}}$ \\
Evolution favorable & $3 / 8$ & $7 / 9$ \\
Évolution & $1 \mathrm{Sy}$. Grêle court avec APT & 1 malabsorption et retard \\
compliquée & 1 malabsorption avec retard staturopondéral et diff. scolaires & staturopondéral \\
Décès & 3 & 1 DCIU 34 SA \\
\hline
\end{tabular}

DCIU, décès in utero ; délai < AET, délai en jours avant alimentation entérale totale ; APT, alimentation parentérale totale définitive.

${ }^{\text {a }}$ Ces chiffres concernent la durée moyenne pour les cinq enfants vivants.

${ }^{\text {b }}$ Ces chiffres concernent la durée moyenne pour les huit enfants vivants.

En cas d'oligohydramnios, on observe également une augmentation de la durée d'alimentation parentérale (70 versus 28) et un séjour hospitalier plus long (95 jours versus 52) par rapport aux patientes chez qui la quantité de liquide était estimée normale.

De même, les enfants chez qui l'échographie anténatale avait mis en évidence des signes de souffrance digestive (dilatation gastrique ou intestinale, épaississement des parois, diminution de la vascularisation mésentérique, 
diminution de la péristaltique, hyperéchogénicité intestinale ou du liquide amniotique) ont également un délai avant alimentation entérale totale et un séjour hospitalier plus long ainsi qu'un pronostic plus péjoratif qu'en cas d'échographie non compliquée (Tableaux 2 et 3).

Le taux de survie à un an pour les enfants nés vivants est de $86 \%$. Le taux de survie sans handicap corrélé au laparoschisis est de $84 \%$.

\section{DISCUSSION}

\section{Associations malformatives}

Il existe des divergences dans la littérature concernant la prévalence, classiquement basse, des malformations associées au laparoschisis.

Les études de Stoll et al. [18] et de Barrisic et al. [19] basées sur des registres de malformations congénitales mettent en évidence des taux non négligeables d'associations malformatives et syndromiques en cas de laparoschisis.

Dans une revue de la littérature portant sur 764 cas de nouveau-nés porteurs d'un laparoschisis, Poulain et al. [20] met en évidence des chiffres plus modestes (8,6 \% de malformations et 0,6 \% de dyschromosomies).

Dans notre série aucune malformation extradigestive n'a été diagnostiquée en prénatal. Trois communications interauriculaires sans répercussion fonctionnelle et une association probablement fortuite avec un syndrome du X fragile ont été décelées après la naissance. Notre faible taux de malformations associées s'explique sans doute en partie par le fait que nous n'avons pas pris en compte les lésions digestives (diverticule de Meckel, malrotation, ischémie digestive et atrésie) qui sont étroitement liées aux mécanismes embryologiques et aux conséquences du laparoschisis.

Les principaux résultats de ces études et la comparaison avec nos données sont résumés dans le Tableau 1.

\section{Évolution de la grossesse et facteurs pronostiques}

En cas de laparoshisis l'objectif du diagnostic anténatal est de confirmer l'absence de malformations associées et de déterminer les facteurs pronostiques qui vont influencer l'évolution de la grossesse.

Ce bilan est évidemment indispensable pour l'information éclairée des parents et la bonne orientation d'une conduite dans la prise en charge anté- et périnatale.

La réalisation systématique d'un caryotype reste discutée. L'incidence d'anomalies chromosomiques n'est pas négligeable (0,6 à $2 \%$ ) et les parents confrontés à l'anxiété générée par la malformation sont souvent demandeurs de cet examen dont le résultat anormal pourrait influencer leur décision quant à la poursuite de la grossesse [21]. Les 19 caryotypes obtenus dans cette série se sont révélés normaux, l'examen a été refusé par un couple et un échec de culture a été enregistré. Les trois derniers cas correspondent à deux diagnostics tardifs et une patiente ne s'étant présenté qu'à une consultation prénatale.

Le succès de la cure chirurgicale et l'évolution à long terme de la fonction digestive sont directement corrélés à l'importance des lésions intestinales constituées in utero et de leurs complications potentielles (atrésie, obstruction intestinale, perforation).

En cas de périviscérite sévère, la réintégration des anses intestinales en un temps dans la cavité abdominale ne pourrait se faire qu'au prix d'une augmentation délétère de la pression intra-abdominale. Les phénomènes ischémiques sont alors renforcés et peuvent conduire à allonger la durée d'alimentation parentérale et parfois compromettre le pronostic vital.

Il est donc judicieux de tenter de définir des critères prédictifs de ces complications dont l'aggravation en fin de grossesse justifie pour Dixon un accouchement prématuré.

La question de facteurs pronostiques identifiables en échographie fait toujours l'objet de discussions. Les deux paramètres le plus souvent cités dans la littérature sont la dilatation de la lumière et l'épaississement de la paroi intestinale [22-25]. 
Une dilatation de la lumière de l'intestin grêle supérieure à 17 mm est associée à une augmentation de la morbidité postnatale avec une spécificité de $75 \%$ et une valeur prédictive positive de 55 \% (risque accru de résection intestinale, allongement de la durée d'alimentation parentérale, hypomotilité et malabsorption persistante).

Abuhamad et Mari estiment qu'une dilatation intestinale supérieure à 10 mm entre 28 et 32 semaines d'aménorrhée est le meilleur critère d'une évolution postnatale compliquée.

Pour Aina-Mumuney [26], une dilatation gastrique est également un facteur pronostique péjoratif accompagné d'une augmentation du risque de décès néonatal, de l'allongement de la durée d'alimentation parentérale et du séjour hospitalier.

L'épaisseur de la paroi intestinale égale ou supérieure à $3 \mathrm{~mm}$ est un critère souvent mentionné mais non validé dans la littérature récente.

L'échographie doppler de l'artère mésentérique supérieure est un outil intéressant pour la mise en évidence d'ischémie mésentérique ou de compressions vasculaires au niveau du collet de la malformation. Les travaux d'Abuhamad et al. [27] n'ont cependant pas pu trouver de différence entre les index de pulsatilité de l'artère mésentérique supérieure des enfants de bon ou de mauvais pronostic contrairement à Luton et al. [28] qui ont démontré une corrélation significative entre ces index, la durée du séjour en néonatologie et la dilatation maximale des anses digestives.

Nous avons comparé l'évolution postnatale des cas de laparoschisis ayant présenté au moins un signe échographique péjoratif (dilatations des anses intestinales ou de l'estomac, épaississement des parois digestives, hyperécho-génicité digestive ou du liquide amniotique, diminution de la vascularisation mésentérique ou de la péristaltique) et les cas avec échographie non compliquée en considérant au moins trois échographies à partir du diagnostic. En présence de signes de complication digestive les résultats montrent une nette augmentation du délai avant la reprise d'une alimentation entérale totale (103 versus 35 jours) et de la durée du séjour hospitalier (127 versus 53 jours). Le pronostic de ces huit enfants est également moins favorable : trois enfants sont décédés dans les trois premiers mois, un enfant reste en alimentation parentérale totale à six mois et un enfant présente un retard staturo-pondéral sur malabsorption et difficultés scolaires à huit ans. Parmi les neuf enfants sans signe échographique de complication digestive sept ont évolué sans complication, un enfant présente un retard staturopondéral à quatre ans et on note un décès in utero à 34 semaines (Tableau 2).

Le retard de croissance intra-utérin (RCIU) est fréquemment observé et reflète un certain degré de souffrance fœtale chronique (60\% dans la revue de Luton et 42,5\% dans sa série personnelle) mais difficilement appréciable en anténatal par la mesure de la circonférence abdominale en raison de l'extériorisation de son contenu. Pour Pramod [29], l'association d'un RCIU au laparoschisis n'affecte pas l'évolution de ces enfants en termes de délai avant alimentation entérale, durée du séjour hospitalier et possibilité de cure chirurgicale en un temps. Dans notre série trois RCIU ont été diagnostiqués en fin de grossesse et quatre enfants (18\%) ont un poids de naissance inférieur au percentile 10. L'évolution de ces enfants a été favorable avec une durée d'alimentation parentérale et de séjour hospitalier comparable aux autres enfants. Les raisons d'un petit poids pour l'âge gestationnel doivent être comprises dans le contexte de la malformation.

Il existe chez les nouveau-nés porteurs d'un laparoschisis une diminution sensible du taux de protéines sériques (51 pour $62 \mathrm{~g} / \mathrm{l}$. dans le groupe témoin) et inversement une augmentation de la concentration des protéines dans le liquide amniotique [30]. Ces observations constituent un élément d'explication du retard de croissance par la transsudation des protéines à travers la paroi intestinale herniée. Dixon fournit un argument supplémentaire en remarquant que le retard de croissance est significativement moins prononcé quand il existe une atrésie intestinale associée au laparoschisis. La perte protéique est conditionnée par la surface d'échange avec le liquide amniotique et par les apports vasculaires de ces protéines au niveau du réseau mésentérique. Le phénomène de drainage des protéines sériques vers le compartiment amniotique est donc maximal en cas d'intestin intact et diminué en cas d'atrésie qui offre une surface d'échange et des apports vasculaires moindres. Pour Luton, le retard de croissance s'explique aussi en cas de périviscérite sévère par l'hypercatabolisme que génère la réaction inflammatoire.

Les anomalies du liquide amniotique sont également fréquemment observées. Un oligoamnios de sévérité variable est observé dans 10 à 40\% des cas. Nous avons comparé l'évolution postnatale des cas de laparoschisis ayant présenté un oligoahydramnios et les cas avec liquide amniotique normal en considérant au moins trois échographies à partir du diagnostic (Tableau 3). Les enfants des patientes ayant présenté un oligoamnios ont un 
délai avant l'alimentation entérale totale et un séjour hospitalier plus long (70 et 95 jours) par rapport aux patientes chez qui la quantité de liquide était estimée normale (28 et 52 jours). L'excès de liquide est moins fréquent et peut être associé à des atrésies ou des sténoses digestives hautes. Un cas de polyhydramnios s'est présenté pour un enfant eutrophique né à terme et dont l'évolution a été simple.

Le développement d'une périviscérite, essentiellement durant le troisième trimestre, peut être empêché ou ralenti par la réalisation d'amnio-infusions (ou d'amnioéchange) qui pour certains cas apparaissent comme une alternative intéressante à l'accouchement prématuré et ses complications potentielles.

Tableau 4 : Données des cinq fotus porteurs d'un laparoschisis traités par amnio-infusion Data for five fetuses with gastroschis treated by amnio-infusion

\begin{tabular}{|c|c|c|c|c|c|c|c|c|}
\hline $\begin{array}{c}\text { AG } \\
\text { diagnostic }\end{array}$ & $\begin{array}{c}\text { AG } 1 \\
\text { AI }\end{array}$ & $\begin{array}{c}\text { Nbre } \\
\text { AI }\end{array}$ & $\begin{array}{c}\text { AG } \\
\text { Naiss. }\end{array}$ & $\begin{array}{l}\text { Voie } \\
\text { Ace. }\end{array}$ & $\begin{array}{c}\text { Aspect du } \\
\text { TD à la naissance }\end{array}$ & $\begin{array}{c}\text { Chirurgie } \\
\text { Primaire } \\
\text { initiale }\end{array}$ & $\begin{array}{c}\text { Durée } \\
\text { AP/jours }\end{array}$ & $\begin{array}{c}\text { Durée } \\
\text { Hospit./jours }\end{array}$ \\
\hline 22 & 35 & 1 & 38 & $\mathrm{C} / \mathrm{S}$ & $\begin{array}{l}\text { Extériorisation grêle, } \\
\text { estomac, foie }\end{array}$ & Non & Décès jour 2 & \\
\hline 18 & 18 & 4 & 31 & c/s & $\begin{array}{l}\text { Nécrose grêle étendue, } \\
\text { collet serré }\end{array}$ & Oui & $>180$ & $>180$ \\
\hline 22 & 28 & 4 & 34 & $\mathrm{C} / \mathrm{S}$ & Absence de périviscérite & Oui & 15 & 21 \\
\hline 28 & 30 & 2 & 34 & $\mathbf{c} / \mathbf{s}$ & Absence de périviscérite & Oui & 53 & 58 \\
\hline 22 & 24 & 3 & 34 & $\mathbf{c} / \mathbf{s}$ & Absence de périviscérite & Oui & 25 & 30 \\
\hline
\end{tabular}

AG, âge gestationnel ; Al, amnio-infusion; TD, tube digestif; AP, alimentation parentérale totale; MFIU, mort fœtale in utero; C/S, césarienne.

Plusieurs équipes [31 33] ont publié des résultats montrant une diminution de la périviscérite ainsi qu'un raccourcissement de la durée de curarisation, du délai d'alimentation orale et du séjour en néonatologie par la réalisation d'amnio-infusions ou d'amnioéchange.

Dans notre série, nous avons réservé l'indication d'amnio-infusion aux cinq cas de laparoschisis présentant un oligohydramnios entre 2000 et 2003. Quatorze amnio-infusions d'un volume moyen de $200 \mathrm{ml}$ de liquide physiologique à température corporelle ont été réalisées en respectant un intervalle de deux ou trois semaines entre chaque infusion. Les principales données sont résumées dans le Tableau 4. Un enfant qui présentait des signes échographiques de complication digestive (épaississement des parois, dilatation gastrique et liquide amniotique échogène) a bénéficié d'une seule infusion à 35 semaines. Il présentait à la naissance un laparoschisis massif (extériorisation de l'estomac, du duodénum, du grêle et d'une partie du foie) et des lésions ischémiques au niveau du foie et de l'intestin. Opéré par la technique du silo, il est décédé au jour 2 dans un tableau d'insuffisance rénale aiguë et de coagulation intravasculaire disséminée. Le deuxième, en oligohydramnios dès 18 semaines et a bénéficié de quatre infusions. Il présentait également des signes échographiques de complication digestive (hyperéchogénicité des anses herniées, dilatation des anses intra-abdominales et faible vascularisation mésentérique). À la naissance, l'intestin grêle d'aspect momifié par compression ischémique au niveau du collet a été l'objet d'une résection quasi complète ne laissant que $11 \mathrm{~cm}$ de jéjunum et obligeant la persistance d'une alimentation parentérale totale à l'âge de six mois. Pour les trois autres, dont un seul présentait un signe échographique de complication digestive (faible vascularisation mésentérique), neuf amnio-infusions ont été réalisées. Dans les trois cas, l'aspect des anses à la naissance ne montrait aucun signe de périviscérite et les enfants ont pu être traités par cure chirurgicale primaire. L'évolution postopératoire a été favorable avec un délai avant alimentation entérale totale (29 jours.) et un séjour hospitalier nettement moins long (34 jours) que la moyenne (63 et 75 jours respectivement). Cette série met en évidence l'intérêt de l'amnio-infusion dans la prévention d'une réaction inflammatoire périviscérale et ses conséquences sur l'évolution postnatale ainsi que l'absence d'effet positif en cas de lésion ischémique constituée en accord les différentes modélisations animales.

\section{Modalité et terme d'accouchement}

En cas de laparoschisis, le moment et la voie d'accouchement restent sujets à controverse. Certains auteurs comme Dixon, qui observe jusqu'à 12,5\% de mortalité périnatale, recommande un accouchement à 35 semaines. L'évitement de l'aggravation tardive des lésions digestives serait préférable aux complications potentielles de la prématurité. Dans un essai prospectif récent mais limité, Moir et al. [34] démontrent l'intérêt d'une extraction prématurée dans une population sélectionnée sur base de l'apparition de signes échographiques de lésions digestives. D'autres auteurs montrent au contraire un allongement de la durée d'alimentation parentérale et du séjour hospitalier ainsi qu'une plus faible proportion de cure chirurgicale en un temps pour les enfants nés prématurément. Ils conseillent un accouchement entre 37 et 38 semaines en utilisant la césarienne uniquement pour des raisons obstétricales [35, 36]. Dans notre série, la durée d'alimentation parentérale et du séjour 
hospitalier s'accroît avec la précocité du terme (Tableau 5). Il est cependant intéressant de constater que dans le groupe des sept enfants nés avant 35 semaines deux enfants qui ont subi des résections importantes de l'intestin grêle pour des lésions ischémiques constatées lors de l'accouchement contribuent principalement à l'allongement de la durée d'alimentation parentérale et du séjour hospitalier. En revanche, dans ce même groupe quatre enfants nés entre 32 et 34 semaines et n'ayant pas présenté de signes échographiques de complication digestive ont des durées d'alimentation parentérale et de séjour hospitalier (33 et 44 jours) comparables aux enfants nés après37 semaines (32 et 38 jours). Trois de ces quatre enfants ont pu bénéficier d'une cure chirurgicale en un temps.

Tableau 5 : Comparaison de l'évolution postnatale en fonction du terme de naissance Postnatal outcome and term at birth

\begin{tabular}{lccc}
\hline & $\begin{array}{c}\text { Avant 35 semaines } \\
(\boldsymbol{n}=\mathbf{7})\end{array}$ & $\begin{array}{c}\text { Entre 35 et 37 semaines } \\
(\boldsymbol{n}=\mathbf{1 0})\end{array}$ & $\begin{array}{c}\text { Après 37 semaines } \\
(\boldsymbol{n}=\mathbf{5})\end{array}$ \\
\hline Interventions & $12(1,7)$ & $21(2,3)$ & $7(1,4)$ \\
Délai < AET & 74 & 55 & 32 \\
Durée séjour & 87 & 77 & 38 \\
\hline
\end{tabular}

Pour certains, la césarienne permettrait d'éviter la contamination des anses intestinales par la flore vaginale, les lésions ischémiques secondaires à la compression du mésentère et le traumatisme intestinal durant le travail. Ces arguments n'ont pas été confirmés par la méta-analyse de Segel et al. [37] qui n'ont pas montré d'avantage de la césarienne par rapport à la voie basse en termes de morbidité, de mortalité, de durée d'alimentation parentéral et d'hospitalisation. Dans notre série 11 enfants sur 22 sont nés par césarienne. Il y a eu sept césariennes électives dont cinq réalisées entre 34 et 36 semaines, parmi celles-ci, quatre ont été réalisées entre 2001 et 2003 dans l'objectif d'éviter l'aggravation tardive des lésions digestives et une en 1993 pour laparoschisis associé à un retard de croissance, les deux dernières pour indication obstétricale (présentation du siège et grossesse gémellaire avec retard de croissance). Quatre césariennes ont été réalisées en cours de travail pour altération des paramètres du monitorage fœtal.

\section{Évolution postnatale et devenir sans morbidité}

Le taux de survie est passé de 20\% en 1960 à 85-90\% actuellement [38]. Dans notre série, parmi les 22 enfants opérés, trois sont décédés dans les trois premiers mois. Nous avons un recul moyen de deux ans et demi pour 17 des 19 enfants vivants. Un enfant de dix ans présente un retard mental lié à un syndrome du X fragile sans rapport avec la malformation en cause. Trois enfants présentent un retard staturopondéral sur malabsorption ou syndrome du grêle court dont un avec des difficultés scolaires à l'âge de huit ans et demi. Parmi ceux-ci un enfant reste en alimentation parentérale totale à son retour au domicile après une hospitalisation de six mois.

\section{CONCLUSION}

Le laparoschisis nécessite une prise en charge dans des centres de référence adaptés. Le diagnostic anténatal est la pierre d'angle de la concertation multidisciplinaire et permet de fournir une information la plus précise possible aux parents.

En cas de malformation isolée, l'enjeu du diagnostic anténatal est de préciser les facteurs pronostiques qui vont influencer la prise en charge périnatale. La difficulté d'interprétation des résultats contradictoires des études évaluant l'effet du terme d'accouchement sur l'évolution postnatale est à mettre en rapport avec l'hétérogénéité des populations étudiées. Dans notre série, les enfants nés avant terme qui ont un séjour hospitalier plus long et une morbidité plus importante sont également ceux qui souffrent d'importantes lésions d'ischémie digestive responsables de l'évolution compliquée. Molik et al. [39] ont d'ailleurs clairement défini deux catégories, simple et complexe, parmi la population d'enfants porteurs d'un laparoschisis selon la présence ou non d'atrésie, de perforation, sténose ou ischémie intestinale constatés à la naissance. Les cas complexes présentent une morbidité et une mortalité nettement accrues, un allongement de la durée d'alimentation parentérale et un séjour hospitalier trois fois plus long que pour les cas simples. Cette catégorisation du pronostic sur base du constat postnatal de lésions digestives sévères n'est actuellement pas accessible au diagnostic anténatal. La multiplicité des mécanismes responsables des lésions digestives permet d'expliquer l'hétérogénéité au sein d'une population d'enfants porteurs d'un laparoschisis et conduit à refuser une conduite à tenir univoque de prise en charge anténatale. Le problème ne se pose donc pas en termes du choix d'une politique systématique d'une voie ou d'un terme d'accouchement mais plutôt dans la difficulté de différencier les enfants qui pourront bénéficier d'un traitement préventif par amnio-infusions ou par accouchement prématuré. Dans cette optique, nous recommandons la réalisation d'amnioéchange qui empêche ou ralentit le développement d'une périviscérite et permettrait également, en déterminant les taux amniotiques de ferritine et de protéines, d'identifier des facteurs 
pronostiques intéressants [40]. Nous recommandons également une surveillance échographique bihebdomadaire jusqu'à 32 semaines et hebdomadaire ensuite. À ce stade, les patientes doivent bénéficier d'une cure de maturation pulmonaire par bêtaméthasone. L'examen doit inclure une évaluation de la croissance fœtale ainsi qu'un doppler de l'artère ombilicale ou d'autres vaisseaux si indiqués. Une évaluation de l'intestin fœtal et du liquide amniotique doit également être réalisée. Une surveillance régulière par cardiotocographie fœtale est souhaitable et permettrait de réduire le risque de décès in utero [41]. Un accouchement prématuré peut être envisagé en cas de persistance ou d'aggravation de signes de complications digestive. Nous réservons la césarienne aux seules indications obstétricales. La définition de critères échographiques stricts demeure problématique. Pour Moïr, au moins trois des quatre critères suivants doivent être rencontrés : dilatation digestive supérieure à $10 \mathrm{~mm}$, épaisseur de la paroi supérieure à $2 \mathrm{~mm}$, diminution de la péristaltique et démonstration échographique d'une périviscérite. D'autres critères doivent également être évalués comme la survenue d'une dilatation gastrique ou l'altération de la vascularisation des anses herniées. En cas de surveillance normale, l'accouchement peut être réalisé à partir de 36 semaines.

Le pronostic de ces enfants s'est fortement amélioré et nos résultats sont en accord avec ceux de la littérature actuelle. La rareté de la pathologie (deux à trois cas par an dans notre centre) nous amène à considérer l'intérêt de la mise en place de collaborations multicentriques afin de pouvoir évaluer dans des conditions standardisées les protocoles de surveillance et de prise en charge anténatale. Il convient, pour terminer, de souligner la complexité de la prise en charge postnatale, la durée d'hospitalisation et la survenue de complications qui peuvent grever l'évolution à plus long terme pour certains d'entre eux.

\section{Références}

[1] Torfs CP, Velie EM, Oechsli FW, et al. A population based study of gastroschisis: demographic, pregnancy and lifestyle factors. Teratology 1994;50:44-53.

[2] Luton M, De Lagausie P, Guibourdenche J, et al. Prognostic factors of prenatally diagnosed gastroschisis. Fetal Diagn Ther 1997;12:714.

[3] De Vries PA. The pathogenesiss of gastroschisis and omphalocele. J Pediatr Surg 1980;15:245-51.

[4] Tibboel D, Raine P, McNee A, et al. Developemental aspects of gastroshisis. J Pediatr Surg 1986;21:865-9.

[5] Hoyme HE, Higginbottom MC, Jones KL. The vascular pathogenesis of gastroschisis: intrauterine interruption of the omphalomesenteric artery. J Pediatr 1981;98:228-31.

[6] Drongowski RA, Smith RK, Coran AG, et al. Contribution of demographic and environemental factors to the etiology of gastroschisis: a hypothesis. Fetal diagn and therap $1991 ; 6: 14-27$.

[7] Dixon JC, Penman DM, Soothill PW. The influence of bowel atresia in gastroschisis on fetal growth, cardiotocograph abnormalities and amniotic fluid staining. Brit J Obs Gyn 2000;107:472-5.

[8] Tibboel D, Raine P, McNee A, et al. Developemental aspects of gastroshisis. J Pediatr Surg 1986;21:865-9.

[9] Langer JC, Longaker MT, Crombleholme TM, et al. Etiology of intestinal damage in gastroschisis. 1 : Effects of amniotic exposure and bowel constriction in a fetal lamb model. J Pediatr Surg 1989;24:992-7.

[10] Kluck P, Tibboel D, Van Der Kamp AWM, et al. The effect of fetal urine on the developpement in gastroschisis. J Pediatr Surg $1983 ; 18: 47-50$.

[11] Aktug T, Ucan B, Olguner M, et al. Amnio-allantoic fluid exchange for prevention of intestinal damage in gastroschisis II: Effects of exchange by using two differents solutions. Eur J Pediatr Surg 1998;8:308-11.

[12] Aktug T, Ucan B, Olguner M, et al. Amnio-allantoic fluid exchange for prevention of intestinal damage in gastroschisis. III: Determination of the waste products removed by exchange. Eur J Pediatr Surg 1998;8:326-8.

[13] Api A, Olguner M, Hakgüder G, et al. Intestinal damage in gastroschisis correlates with the concentration of intraamniotic meconium. J Pediatr Surg 2001;36:1811-5.

[14] Akgür FM, Özdemir T, Olguner M, et al. An experimental study investitating the effects of intraperitoneal human neonatal urine and meconium on rat intestines. Res Exp Med (Berl) 1998;198:207-13.

[15] Morrison JJ, Klein N, Chitty L, et al. Intra-amniotic inflammation in human gastroschisis: a possible aetiology of postnatal bowel 
Published in : Journal de Gynécologie, Obstétrique et Biologie de la Reproduction (2007), vol. 36, iss. 5, pp 486-95

Status : Postprint (Author's version)

dysfunction. Br J Obstet Gynaecol 1998;105:1200-4.

[16] Mahieu-Caputo D, Muller F, Jouvet PH, et al. Amniotic fluid B endorphin : a prognostic marker for gastroschisis? J Pediatr Surg 2002;37:1602-6.

[17] Luton D, De Lagausie P, Guibourdenche J, et al. Pognostic factors of prenatally diagnosed gastrschisis. Fetal Diagn Ther 1997;12:7-14.

[18] Stoll C, Alembik Y, Dott B, et al. Risk factors in abdominal wall defects (omphalocele and gastroschisis): a study in a serie of 265,858 consecutive birth. Ann Genet 2001:201-8.

[19] Barrisic I, Clementi M, Häusler M, et al. Evalution of prenatal diagnosis of fetal abdominal wall defects by 19 European registries. Ultrasound Obstet Gynecol 2001;18:309-16.

[20] Poulain P, Milon J, Frémont B, et al. Remarks about the prognosis in case of antenatal diagnosis of gastroschisis. Eur J Obstet Gynecol Reprod Biol 1994:185-90.

[21] Robinson JN, Abuhamad AZ. Abdominal wall and umbilical cord anomalies. Clin Perinatol 2000;27:947-78.

[22] Langer JC, Khanna J, Caco C, et al. Prenatal diagnosis of gastroschisis: development of objective sonographic criteria for predicting outcome. Obstet Gynecol 1993;81:53-6.

[23] Luton M, De Lagausie P, Guibourdenche J, et al. Prognostic factors of prenatally diagnosed gastroschisis. Fetal Diagn Ther 1997;12:714.

[24] Pryde P, Bardicef M, Treadwell MC, et al. Gastroschisis: can antenatal ultrasound predict outcome? Obstet Gynecol 1994; 84:505-9.

[25] Brun M, Grignon A, Guibaud L, et al. Gastroschisis: are prenatal ultrasonographic findings usefull for assessing the prognosis? Pediatr Radiol 1996;26:723-6.

[26] Aina-Mumuney AJ, Fisher AC, Blakemore KJ, et al. A dilated fetal stomach predicts a complicated postnatal course in case of prenatally diagnosed gastroschisis. Am J Obstet Gynecol 2004;190:1326-30.

[27] Abuhamad AZ, Mari G, Cortina RM, et al. Superior mesenteric artery doppler velocimetry and ultrasonographic assessment of fetal bowel: a prospective longitudinal study. Am J Obstet Gynecol 1997;176:985-90.

[28] Luton D, Guibourdenche J, Vuillard E, et al. Prenatal management of gastroschisis : the place of the amnioexchange procedure. Clin Perinatol 2003;30:551-72.

[29] Puligandla SP, Janvier A, Laberge JM, et al. The significance of intrauterine growth restriction is different from prematurity for the outcome of infants with gastroschisis. J Pediatr Surg 2004;39:1200-4.

[30] Caroll SG, Ping-yi K, Kyle MP, et al. Fetal protein loss in gastroschisis as an explanation of associated morbidity. Am J Obstet Gynecol $2001 ; 184: 1297-301$.

[31] Dommergues M, Ansker Y, Aubry MC, et al. Serial transabdominal amnioinfusion in the management of gastroschisis with severe olygohydramnios. J Pediatr Surg 1996;31:1297-9.

[32] Sapin E, Mahieu D, Borgnon J, et al. Transabdominal amnioinfusion to avoid fetal demise and intestinal damage in fetuses with gastroschisis and severe oligohydramnios. J Pediatr Surg 2000;35:598-600.

[33] Aktug T, Demir N, Akgur FM, et al. Pretreatment of gastroschisis with transabdominal amniotic fluid exchange. Obstet Gynecol $1998 ; 91: 821-3$

[34] Moir CR, Ramsey PS, Ogburn PL, et al. A prospective trial of elective preterm delivery for fetal gastroschisis. Am J Perinatol 2004;21:289-94.

[35] Driver CP, Bruce J, Bianchi A, et al. The contemporary outcome of gastroschisis. J Pediatr Surg 2000;35:1719-23.

[36] Huang J, Kurkchubasche AG, Luks FI, et al. Benefits of term delivery in infants with antenatally diagnosed gastroschisis. Obstet Gynecol 2002;100:695-9.

[37] Segel SY, Marder SJ, Parry S, et al. Fetal abdominal wall defects and mode of delivery : A systematic review. ACOG 2001;98:867-73.

[38] Babcook CJ, Hedrick MH, Goldstein RB, et al. Gastroschisis: can the sonography of the fetal bowel accurately predict postnatal outcome? J Ultrasound Med 1994;13:701-6.

[39] Molik KA, Gingalewski CA, West FJ, et al. Gastroschisis: a plea for risk categorization. J Pediatr Surg 2001;36:51-5. 
Published in : Journal de Gynécologie, Obstétrique et Biologie de la Reproduction (2007), vol. 36, iss. 5, pp 486-95 Status : Postprint (Author's version)

[40] Burk L, Volumenie JL, de Lagausie P, et al. Amniotic fluid inflammatory proteins and digestive compounds profile in fetuses with gastroschisis undergoing amnioexchange. BJOG 2004;111:292-7.

[41] Brantberg A, Blaas HGK, Salvesen KA, et al. Surveillance and outcome of fetuses with gastroschisis. Ultrasound Obstet Gynecol 2004;23:4-13. 